\title{
CONCENTRACIÓN DE LAS MYPES Y SU IMPACTO EN EL CRECIMIENTO ECONÓMICO
}

\author{
MYPES CONCENTRATION AND ITS IMPACT ON ECONOMIC GROWTH \\ Nicko Alberto Gomero Gonzales* \\ Docente Principal de la Facultad de Ciencias Contables \\ Universidad Nacional Mayor de San Marcos-UNMSM / Lima-Perú \\ [Recepción: Marzo de 2015/ Conformidad: Abril 2015]
}

\section{RESUMEN}

Como es natural, las empresas buscan rentabilizar sus operaciones; para ello, buscan espacios económicos que las conlleve asegurar mercado, reducir costos, realizar reconversión tecnológica, medios que los conducirá a potenciar su competitividad y asegurar su sostenibilidad. Basado en este criterio es que las MYPES muestran su preferencia de localización en las zonas metropolitanas, especialmente la región Lima y Callao, que agrupa en promedio el $51.0 \%$ de las micro y pequeñas empresas formales en contraste con la franja económica de la sierra, que agrupa al $21.4 \%$ de este tipo de organizaciones, cifra que se agrava cuando se aprecia la cantidad de empresas de la selva en donde se concentra solo el $6.2 \%$ del conglomerado empresarial. Estos resultados se van a reflejar en la calidad de vida de la población, el cual fue uno de los objetivos de la investigación, en donde se evidencia una relación estrecha entre los resultados económicos, producto del accionar de las empresas y los indicadores de calidad de vida. Bajo este criterio se establece que, donde exista menor cantidad de empresas, como son las micro y pequeñas empresas, mayor será la probabilidad de caer en desequilibrios sociales.

\section{Palabras clave:}

Concentración de empresas; MYPES; mercado; costos; desequilibrios sociales; calidad de vida.

\begin{abstract}
Naturally, companies seek profitable operations; to this end, they pursue economic spaces that ensure market leading, costs reduction, technological adaptation, means that lead to enhance their competitiveness and ensure its sustainability. Based on this criterion, MYPES show a preference for location in metropolitan areas, especially Lima and Callao region, which include an average of $51.0 \%$ of micro and small enterprises in contrast to the formal mountain economic fringe, that gathers $21.4 \%$ of these organization, an amount aggravated when we observe that the number of the forest companies where the concentration is only $6.2 \%$ of the business conglomeration. These results will be reflected in the quality of life of the population, which was one of the goals of the research, where a close relationship among economic performance, the product of the actions of the companies and of quality of life indicators. Under this criterion we state that, where fewer companies exist, such as micro and small enterprises, the greater the probability of falling into social imbalances.
\end{abstract}

\section{Keywords:}

Companies concentration; MYPES; market; costs; social imbalances; quality of life.

\footnotetext{
* Doctor en Ciencias Económicas. Magíster en Contabilidad con mención en Banca y Finanzas-UNMSM. Email: gomero_econ@yahoo.es
} 


\section{INTRODUCCIÓN}

Las pequeñas y microempresas, a pesar de sus deficiencias estructurales han jugado un papel importante en el proceso de crecimiento económico del país. Sus procesos productivos aún tienen mucho que mejorar para responder a las exigencias del mercado, estas organizaciones han sabido abrirse paso en un terreno lleno de dificultades, como de las aperturas de los mercados y los TLC. Bajo estos escenarios turbulentos han sabido ganar fortalezas y convertirse en importantes aportantes a la formación del PBI, por cierto muy por debajo de lo que aporta el conglomerado de las grandes capitales.

Las MYPES, han sabido entrar a competir en mercados externos, no masificadamente, pero si han dado señales que también pueden entrar a estos mercados, con productos diferenciados y con elevada demanda potencial. Hay que tener en cuenta que la generación de divisas recae en las grandes empresas, especialmente, en aquellas que operan en el sector minero.

La informalidad es uno de los problemas que el gobierno no ha podido dar solución, las cifras señalan que estas, inclusive se encuentran por encima de las empresas que operan respetando las normas internas. Si bien este tipo de empresas crean escenarios favorables para la captación de mano de obra, pero generan distorsiones severas que en nada contribuyen al proceso de desarrollo. Detrás de esta actividad encontramos al contrabando, que como se conoce se constituye en uno de los más importantes evasores de impuestos. Contrariamente a lo que se presenta en el sector informal, las empresas legalmente constituidas no solo se someten a las reglas del mercado, sino también, al marco normativo gubernamental.

La pobreza está muy ligada a la formación de empresas, presentándose una correlación negativa entre ambas variables, las estadísticas oficiales dan pie a afirmar que en aquellas zonas donde más concentradas están las empresas, los síntomas de la pobreza tiende a disminuir, hecho que se fundamenta en la creación de riqueza y los efectos multiplicadores que generan las inversiones. Por ello, en aquellas regiones donde está ausente la inversión privada o la presencia del Estado a través de proyectos productivos, la pobreza extrema se agudiza, bajando la calidad de vida de la población.

\section{MARCO TEÓRICO}

\section{Las MYPES. Concepto}

Las pequeñas y microempresas son organizaciones producto del emprendimiento de personas que arriesgan pequeños capitales y que se someten a las reglas del mercado. No reciben subsidios, ni beneficios colaterales como si los obtiene las empresas de gran envergadura, específicamente las exportadoras, pero a base de imaginación y destreza muchas de ellas logran obtener posiciones importantes en los segmentos de mercado donde les toca operar. La mayoría de ellas, no siguen una disciplina académica, ni los protocolos económicos o financieros, pero si saben destrabar problemas, que como es natural se presentan a diario en su quehacer económico.

Jorge Arbulú y Juan Jose Otoya $(2005)^{1}$ en la investigación realizada, se lee lo siguiente:

“... La legislación peruana define a la PYME (Pequeña y Micro Empresa) como la unidad económica constituida por una persona natural o jurídica, bajo cualquier forma de organización o gestión empresarial contemplada en la legislación vigente, que tiene como objeto desarrollar actividades de extracción, transformación, producción, comercialización de bienes o prestación de servicios (...) debiendo contar con las siguientes características:

Microempresa:

- Número total de trabajadores de (1 a 10).

- Niveles de venta: menores a 150 UIT

Pequeña empresa:

- Número total de trabajadores con un máximo de 50 - Niveles de venta anuales entre 151 y 850.

\section{Mediana empresa:}

- Ventas anuales superiores a 1700 UIT hasta monto máximo de 2300 UIT"

Las especificaciones que hacen los autores referenciados con respecto a estos tipos de empresas, aclara el concepto sobre los tipos de organización que centrará el análisis de la presente investigación.

1 Tomado de: CENDOC. Esan. edu.pe

30/ QuipuramaVoc | Vol. 23(43) 2015 
El Ministerio de la Producción en su informe: MIPYME ${ }^{2}$. Estadísticas de la micro, pequeña y mediana empresa argumente que: "... para establecer el tamaño empresarial se utiliza la definición establecida en la Ley $\mathrm{N}^{\circ} 30056$, ley que modifica diversas leyes para facilitar la inversión, impulsar el desarrollo productivo y crecimiento empresarial. Esa ley tiene por objeto constituir el marco legal para promocionar la competitividad, la formalización y el desarrollo de las micro, pequeñas y medianas empresas (MIPYMES), mediante el establecimiento de políticas de alcance general y la creación de instrumentos de apoyo y promoción; la incentivación de la inversión privada, la producción, el acceso a los mercados internos y externos, y otras políticas que impulsen el emprendimiento y permitan mejorar la organización empresarial junto con el crecimiento sostenido de estas unidades económicas".

La estructura legal de este tipo de organizaciones y los bajos niveles de capital que utilizan para iniciar sus operaciones hacen posible su masificación y distribución a nivel nacional, pero no están simétricamente localizados, hecho por la cual limita su capacidad apalancadora en el proceso de desarrollo; por ello, los indicadores de pobreza son más notorios en aquellas zonas donde es ajena la inversión privada. El Estado en muchas de estas regiones, asume su responsabilidad, pero por los grados de corrupción que se aprecia en las actividades de ejecución, los efectos sociales son marginales.

Uno de los grandes problemas que tiene que afrontar el gobierno con respecto a este tipo de empresas es la informalidad, que dicho sea de paso en el Perú sobrepasa a la cantidad de organizaciones que operan bajo los parámetros de la formalidad. Las empresas que actúan marginalmente de las normas legales internas, generan externalidades que entorpecen el proceso de desarrollo nacional o regional. No pagan impuestos, venden clandestinamente, pagan a sus trabajadores sin los beneficios laborales, generan competencia desleal, es decir crean una serie de es- cenarios distorsionadores y que dislocan el proceso de desarrollo.

La Organización Internacional del Trabajo (OIT) define a la economía informal como: "el conjunto de actividades económicas desarrolladas por los trabajadores y las unidades económicas que, tanto en la legislación como en la práctica, están insuficientemente contempladas por sistemas formales o no lo están en absoluto. Las actividades de esas personas y empresas no están recogidas por la ley, lo que significa que se desempeñan al margen de ella; o no están contempladas en la práctica, es decir que, si bien estas personas operan dentro del ámbito de la ley, esta no se aplica o no se cumple; o la propia ley no fomenta su cumplimiento por ser inadecuada, engorrosa o imponer costos excesivos."

Guillermo Miranda (2005) $)^{4}$, explica lo siguiente "informalidad laboral a la exclusión total o parcial de las normas que conforman al derecho del trabajo. Este fenómeno se produce en la economía formal e informal, y puede ser conocida desde una aproximación a las unidades de producción (empresa) o a las circunstancias específicas de los trabajadores". Agregando además que: "La informalidad laboral de la economía informal presenta causas aparentemente estructurales al sistema capitalista, el capital se concentra en los países centrales mientras que, los excedentes que se trasladan a la periferia originan que gran parte de las unidades de producción de nuestros países no puedan acceder al sistema. Desde la perspectiva de los trabajadores la economía formal no produce los puestos de trabajo suficientes (en cantidad y calidad), y nuestros desempleados al carecer de una protección social efectiva se sumergen en la informalidad para subsistir en unidades de producción informales o convertirse en trabajadores autónomos".

Como se puede apreciar, estas dos últimas definiciones expresan muy bien lo que es informalidad, dejando entrever que este fenómeno es producto de un gobierno deficiente y la falta de espacios dentro de la economía formal para insertar a la mano de

2 http://www.produce.gob.pe

3 Tomado de www.paradigmas.mx.informalidad

4 Informalidad laboral y la acción estatal en el Perú: Por Guillermo Miranda, Ministerio de Trabajo y Promoción del Empleo de Perú. OEA segunda reunión de grupo de trabajo de la XIII Conferencia Interamericana de Ministros de Trabajo. Buenos Aires Argentina 2005. 
obra excedente, lo que llama exclusión laboral. El surgimiento de la economía informal en si obedece a este último factor señalado, hecho que se evidenció en el Perú, cuando se aplicó una política de ajuste macroeconómico por los años 90 , con resultados sociales impactantes que hizo posible que los trabajadores busquen alternativas de empleo para sobrevivir y lo encontraron en la informalidad, que en el transcurso del tiempo se ha ido sosteniendo con señales de crecimiento. Tarea que le compete al gobierno para su erradicación. Si bien, esta actividad ha generado alternativas de empleo pero ha debilitado las estructuras económicas del país, como lo haría en cualquier economía. No hay que perder de vista que este fenómeno representa más del $50 \%$ de la actividad económica en el mundo 5 .

\section{Gestión económica de las MYPES}

Los emprendimientos son empresas que deben auto sostenerse: para ello, es básico que ejecuten una serie de acciones que les conlleve a maximizar sus beneficios en el peor de los escenarios, evitar la pérdidas continuas. Se necesita una buena gerencia, responsabilidad en el manejo de los recursos, en la visión de mercado, ir mejorando los procesos, todo ello con seguridad se transformará en mayores niveles de competitividad. Si bien, muchos de los micro o pequeños empresarios no han sido calificados en universidades o academias especializadas, pero si han dado muestras de tener conocimiento de negocios, por ello, no es una casualidad que del $100 \%$ de las organizaciones productivas, el $95 \%$ lo encontramos en este segmento empresarial.

Como en todo negocio se tiene que ensayar medidas para aumentar las ventas, a través de un fortalecimiento de su cartera de clientes, el cual pasa como es de entender por encontrar nuevos espacios de mercado. Todos estos son los instrumentales de esta comunidad empresarial que debe utilizar para lograr posicionarse de una porción del mercado.

Las 4 P's del marketing no solo es exclusividad de las grandes compañías. El manejo responsable del precio, plaza, promoción y producto, también están presente en la gestión de estas empresas, que a través de la mezcla comercial pertinente establecen estrategias para lograr mejores posicionamientos de mercado. Hay que tener presente que Jerome McArthy desarrolló la teoría de las 4 P's del marketing, que ahora ha dado paso a las $4 \mathrm{C}$ que son manejados, aunque empíricamente por los gestores de micro negocios. El uso adecuado de las 4C, cliente, costos, convivencia, comunicación, les permite sostenerse en el mercado. Sobre este punto, es importante señalar que no solo hay que preocuparse de los costos, sino también, de los beneficios que se dará al consumidor a través del producto (cliente), y por cierto darle una buena información ( comunicación) y ser sus aliados estratégico (comunicación), son las reglas básicas para la competividad.

Cuando las micro y pequeñas empresas entran al mercado, tienen que enfrentar a una competencia de origen interno y externo. Por ello, tiene que fijar precios que les permita asegurar un mercado e ingresos sostenidos, bajo el objetivo de obtener utilidades y con ello, asegurar nuevas inversiones y una tendencia de crecimiento.

Philip Kotler (2012) cuando explica sobre los 10 pecados capitales del marketing señala: "Uno de los errores más comunes del marketing es intentar vender a todo el mundo. Incluso dentro de un segmento de mercado, nos encontraremos con gente con muy diversas necesidades...”. Este concepto, si bien, no lo pueden conocer los emprendedores, pero si en su quehacer diario lo aplican empíricamente, por ello los conglomerados de negocios agrupados por cada tipo de servicio, saben identificar su mercado y lograr un posicionamiento sostenido.

Así, como se gestiona el mercado y los ingresos, también es pertinente que las Pymes se preocupen en sus costos, que se define como el desembolso monetario para la fabricación de un producto. Cuando se elabora un producto aparecen los costos fijos y variables. Los primeros de ellos tienen que ser asumidos independientemente cualquiera sea la producción, pero cuando se trata de los variables los desembolsos van a aparejada a la producción. Como detrás de los costos están los insumos, la materia prima, mano 
de obra, equipos, es necesario que las unidades de negocios implementen políticas que les conlleven a una óptima gestión de estos recursos, lo cual va a incidir en sus indicadores de rentabilidad.

La inversión, es otro concepto básico que se maneja en el mundo de los negocios, este medio permite que las unidades productivas puedan crecer en el tiempo y con ello, ser más competitivas. La reconversión tecnológica, la capacitación del personal, compra de nuevos equipos, ampliación de planta, son acervos básicos que están presente en todo programa de inversión, el cual como se entiende necesita financiamiento, los cuales provienen del mercado financiero formal e informal.

Los microempresarios siempre están mentalizados en invertir, pero la traba que encuentran es el financiamiento, ya que la tasa de interés que les cargan está por encima de los demás sujetos prestatarios. Pero hay opciones que pueden ser utilizadas, una de ellas es COFIDE que maneja un portafolio de líneas de crédito que podría cubrir las necesidades crediticias de los emprendedores.

\section{RESULTADOS DE LA INVESTIGACIÓN}

Las pequeñas y micro empresas calificadas como organizaciones emergentes se han desarrollado por todo el país. Por ello, es que su grado de participación en la pirámide empresarial llega en promedio al $99 \%{ }^{6}$. La configuración de estos negocios además ha ido acompasada con el desarrollo de un mercado laboral, con un aporte del $40.6 \%$, donde los integrantes de la PEA han visto la oportunidad de mejorar su calidad de vida. Si bien, es cierto que la distribución de las empresas no es equitativa o simétrica pero a pesar de esta debilidad no deja de ser una importante generadora de riqueza en el sector real de la economía.

Como se conoce, la sostenibilidad de la rentabilidad de los negocios, entre un conjunto de factores, depende de su ubicación; específicamente para tomar esta decisión se tiene que evaluar la carga tributaria, el costo de transporte, la dimensión de mercado, elementos claves para lograr este propósito.
Cuando se quiere emprender un negocio, no hay que perder de vista que es sumamente importante la elaboración de un estudio de proyecto o llamado también Plan de Negocios, dentro de este documento uno de los puntos relevantes es la ubicación o localización, que en cierta forma coadyuvarán a cumplir con las metas de rentabilidad del negocio a implementar. Pero cuando se forma una micro empresa, se obvia este paso técnico y se toma la decisión llevado por la intuición o la experiencia de haber laborado en el sector donde ha de funcionar la nueva organización. La mayoría va hacia las metrópolis como es Lima metropolitana.

Tal como se observa, en el cuadro No 1 Lima, centraliza el $47.3 \%$ de las empresas; es decir, de cada 100 empresas formadas a nivel nacional 47 centran sus operaciones en la ciudad capital, y si a esta cifra se le suma las del Callao están llegando en promedio al 50\%. Estas cifras corroboran lo manifestado, que esta región es el gran aportante a la riqueza del país o en la formación del PBI. Esta centralización obedece a factores que en las provincias o departamentos del país no se puede encontrar. Esta centralización, no hay que perder de vista es un alimentador de la pobreza en aquellas regiones que no se ven beneficiados con esta dinámica empresarial. Para corroborar lo manifestado basta apreciar las cifras de Huancavelica, Apurímac, Pasco, Ayacucho cuya concentración de empresas, independientemente cualquiera sea su dimensión, está en un rango del 0,4\% a 1,2\%. De cada 100 empresas solo 1 se ubica en esos espacios geográficos. Por lo tanto, su aporte para corregir los problemas estructurales de la pobreza y la redistribución de la renta es limitada.

Solo Piura, Lambayeque, la libertad, Ica se destacan como zonas que se proyectan para emprender negocios agroindustriales, especialmente aquellos que se dedican a la exportación. Estas zonas se vienen destacando como áreas productivas y un mercado laboral en vías de fortalecimiento. 
Cuadro No 01: Perú: Número de empresas según departamento

\begin{tabular}{|c|c|c|}
\hline Departamento & Absoluto & $\%$ \\
\hline Nacional & 1575529 & 100 \\
\hline Amazonas & 9162 & 0.6 \\
\hline Ancash & 49338 & 3.1 \\
\hline Apurímac & 11868 & 0.8 \\
\hline Arequipa & 87486 & 5.6 \\
\hline Ayacucho & 17880 & 1.1 \\
\hline Cajamarca & 34443 & 2.2 \\
\hline Callao & 54288 & 3.4 \\
\hline Cusco & 54276 & 3.4 \\
\hline Huancavelica & 6555 & 0.4 \\
\hline Huánuco & 21993 & 1.4 \\
\hline Ica & 37653 & 2.4 \\
\hline Junín & 57212 & 3.6 \\
\hline La Libertad & 79362 & 5.0 \\
\hline Lambayeque & 56636 & 3.6 \\
\hline Lima & 745455 & 47.3 \\
\hline Loreto & 31196 & 2.0 \\
\hline Madre De Dios & 11269 & 0.7 \\
\hline Moquegua & 10785 & 0.7 \\
\hline Pasco & 9885 & 0.6 \\
\hline Piura & 65838 & 4.2 \\
\hline Puno & 33532 & 2.1 \\
\hline San Martin & 28437 & 1.8 \\
\hline Tacna & 25403 & 1.6 \\
\hline Tumbes & 12767 & 0.8 \\
\hline Ucayali & 22810 & 1.4 \\
\hline
\end{tabular}

Fuente: INEI.

Esta misma configuración se presenta a nivel de microempresas, las cifras evidencian una fuerte concentración en Lima Metropolitana, cuyo grado de participación llega al 46,8\%. La participación de los demás departamentos se puede considerar como marginal en la estructura empresarial. Entre nueve departamentos, su grado de participación llega al $30 \%$, estas cifras dan una idea sobre la asimetría en la distribución de los micros negocios en el Perú. Huancavelica, sigue siendo el departamento que menos empresas presenta, su aporte en la pirámide de estos negocios solo llega a; $0.4 \%$. Tumbes, Apurímac,
Madre de Dios, Pasco, son otros departamentos que no se han desarrollado sustancialmente en este tipo de negocios y en donde se ha desarrollado en mayor grado los síntomas de la pobreza.

La concentración de los micros negocios en Lima tiene una explicación; por un lado, está el mercado, el tipo de mano de obra, los proveedores, las entidades administrativas del gobierno, infraestructura económica. Todos estos factores ayudan a que se de este resultado, que dicho sea de paso se repite en forma similar en los pequeños negocios, tal como se aprecia en el gráfico $\mathrm{N}^{\circ} 01$ y cuadro $\mathrm{N}^{\circ} 02$. 
Gráfico No 01: Perú: Microempresas por departamentos, setiembre 2012

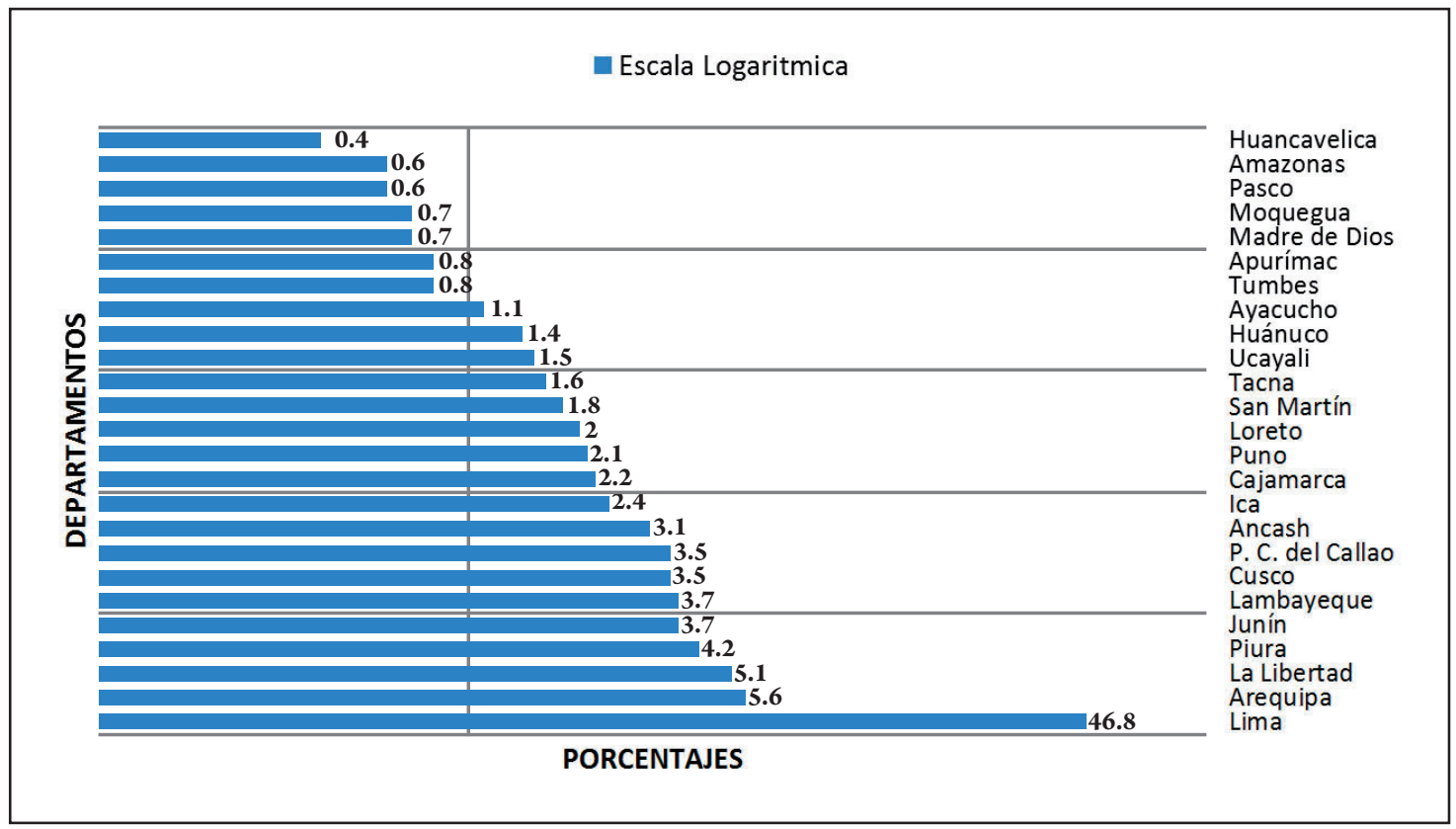

Fuente: Ministerio de la producción (PRODUCE)

A nivel de pequeños negocios, la estructura empresarial es un reflejo de los micronegocios. Lima centraliza el 59.9\% de estos negocios y Huancavelica lo hace con un $0.2 \%$. Presentándose con ello, una perfecta asimetría que además va a sustentar la diferencia en el proceso de crecimiento económico regional. Según las estadísticas de PRODUCE en Lima operan 30025 pequeñas empresas, en Huancavelica solo se aprecia la operatividad de 108 de estos tipos de organizaciones, castigándose con ello en este segundo espacio económico al mercado laboral, fenómeno que va a originar el flujo migratorio, ya que la población de la región no encuentra oportunidades laborales y si los hay, se focalizan en la agricultura, ganadería con salarios de subsistencia.

Las micro y pequeñas empresas cualquiera sea su tipo de estructura organizativa, que como ya se mencionó, concentran sus operaciones en LM, desarrollan sus actividades mayormente en el sector comercio y servicio, seguido de la actividad manufacturera. Las microempresas que se dedican al comercio llegan al 47\%. Las empresas dedicadas a la actividad manufacturera es limitada siendo su participación del $9 \%$. El comercio es la actividad que se ha potenciado en estos últimos años, que conglomera una importante cantidad de mano de obra, específicamente, no calificada. Esta misma cifra se repite a nivel de pequeños negocios que entre las empresas que se dedican al comercio y a la industria manufacturera llega al 52\%.

Estas cifras a nivel de micro y pequeños negocios sobre el comercio evidencian la propensión de las personas por formar empresas que solo realizan actividades de transacción comercial, sin generar valor agregado y contribución efectiva a la formación del PBI. Otra actividad que destaca es la de servicios profesionales, segmento empresarial que se dedica al servicio de asesoría empresarial, su aporte en las microempresas llega al 9.9\%, tal como se observa en el cuadro $\mathrm{N}^{\circ} 03$. Si bien, estas cifras generan espacios laborales e ingresos a favor de los actores económicos, pero su contribución al desarrollo regional es marginal, ya que solo son actividades comerciales con mínimos efectos multiplicadores de tipo económico y social. Por cierto, la contribución al PBI podría ser importante, pero no se constituye en una palanca suficiente para impulsar el desarrollo. 
Cuadro No 02: Perú: Pequeña empresa, según departamento, Setiembre 2012

\begin{tabular}{|c|c|c|}
\hline Departamento & Absoluto & $\%$ \\
\hline Nacional & 61129 & 100 \\
\hline Amazonas & 252 & 0.4 \\
\hline Ancash & 1534 & 2.5 \\
\hline Apurímac & 247 & 0.4 \\
\hline Arequipa & 3004 & 4.9 \\
\hline Ayacucho & 425 & 0.7 \\
\hline Cajamarca & 967 & 1.6 \\
\hline Callao & 1942 & 3.2 \\
\hline Cusco & 1276 & 2.1 \\
\hline Huancavelica & 108 & 0.2 \\
\hline Huánuco & 530 & 0.9 \\
\hline Ica & 1294 & 2.1 \\
\hline Junín & 1324 & 2.2 \\
\hline La Libertad & 2498 & 4.1 \\
\hline Lambayeque & 1380 & 2.3 \\
\hline Lima & 36625 & 59.9 \\
\hline Loreto & 1149 & 1.9 \\
\hline Madre De Dios & 306 & 0.5 \\
\hline Moquegua & 280 & 0.5 \\
\hline Pasco & 296 & 0.5 \\
\hline Piura & 2035 & 3.3 \\
\hline Puno & 841 & 1.4 \\
\hline San Martin & 818 & 1.3 \\
\hline Tacna & 819 & 1.3 \\
\hline Tumbes & 326 & 0.5 \\
\hline Ucayali & 853 & 1.4 \\
\hline
\end{tabular}

Fuente: INEI. 
Cuadro No 03: Perú: Número de microempresas según actividad económica - 2012

\begin{tabular}{|l|r|c|}
\hline \multicolumn{1}{|c|}{ Actividad económica } & Absoluto & \% \\
\hline \multicolumn{1}{|c|}{ Nacional } & $\mathbf{1 4 9 9 1 0 1}$ & $\mathbf{1 0 0}$ \\
\hline Agricultura, ganadería, silvicultura y pesca & 30014 & 2,0 \\
\hline Explotación de minas y canteras & 8450 & 0,6 \\
\hline Industrias manufactureras & 135051 & 9,0 \\
\hline Electricidad, gas y agua & 3385 & 0,2 \\
\hline Construcción & 38468 & 2,6 \\
\hline Comercio y reparación de vehículos automotores y motocicletas & 703199 & 46,9 \\
\hline Transporte y almacenamiento & 68648 & 4,6 \\
\hline Actividades de alojamiento y de servicio de comidas & 122018 & 8,1 \\
\hline Información y comunicaciones & 49907 & 3,3 \\
\hline Servicios profesionales, técnicos y de apoyo empresarial & 148716 & 9,9 \\
\hline Otros servicios 1/ & 191245 & 12.8 \\
\hline $\begin{array}{l}\text { 1/ Incluye financieras, seguros, inmobiliarias, administración pública, enseñanza, salud, artísticas, entrete- } \\
\text { nimiento y otros servicios. }\end{array}$ \\
\hline
\end{tabular}

Fuente: INEI - Directorio Central de Empresas y Establecimientos.

$\mathrm{Si}$, tomamos en cuenta las micro, pequeñas y medianas empresas, denominadas (MIPYMES), también se evidencia que la concentración empresarial se refleja en la formación del PBI; es decir, cuanto más empresas operen en una región, el crecimiento económico tendrá el mismo comportamiento y con ello, paliando los síntomas de la pobreza. Las cifras del Ministerio de la Producción señalan que como Lima y Arequipa concentran 53.5\% de las empresas, siendo su aporte a la formación del PBI del 59.2\%, reflejándose además, estos resultados en la calidad de vida de esta parte de la población. Claro está que existe una total asimetría en la dinámica económica entre estas dos regiones. Pero, si se compara Arequipa con La Libertad, sus aportes son importantes al PBI, pero muy alejados de la ciudad capital que práctica- mente es el sostén del crecimiento económico. Las cifras se muestran en el cuadro $\mathrm{N}^{\circ} 04$.

Como explica el Ministerio de la Producción; "A nivel nacional, las regiones de Lima y del Callao concentran el 51,0\% de las MIPYMES, y el 49,0\% restante, se distribuye a nivel nacional en las regiones que conforman la costa, sierra y selva”.

Todo lo contrario sucede con Huancavelica, Amazonas, Pasco, Moquegua, Madre de Dios, cuyo aporte de estos departamentos solo llega al 3.9\%. Hay que destacar que estos espacios económicos en forma agregada solo centran sus operaciones el 3.7\% de las MIPYMES. Por ello, es que se argumenta que en estas zonas, la población experimenta muchos problemas sociales que inclusive no son cubiertos por la autoridad gubernamental. 
Cuadro No 04: Perú: MIPYMES formales, según regiones, 2012

\begin{tabular}{|l|c|c|c|c|}
\hline \multirow{2}{*}{ Región } & \multicolumn{2}{|c|}{ PBI $^{\mathbf{1}}$} & \multicolumn{2}{c|}{ MIPYMES } \\
\cline { 2 - 5 } & PBI en millones de S/. & \% & Número & \% \\
\hline Lima & 120288 & 53.6 & 640547 & 47.8 \\
\hline Arequipa & 12621 & 5.6 & 76417 & 5.7 \\
\hline La Libertad & 10602 & 4.7 & 70279 & 5.2 \\
\hline Piura & 8922 & 4.0 & 52639 & 3.9 \\
\hline Cusco & 6705 & 3.0 & 50720 & 3.8 \\
\hline Junín & 7001 & 3.1 & 48551 & 3.6 \\
\hline Lambayeque & 6042 & 2.7 & 47066 & 3.5 \\
\hline Callao ${ }^{2}$ & & - & 42608 & 3.2 \\
\hline Ancash & 7407 & 3.3 & 40747 & 3.0 \\
\hline Ica & 6976 & 3.1 & 34154 & 2.5 \\
\hline Cajamarca & 5550 & 2.5 & 28492 & 2.1 \\
\hline Puno & 4722 & 2.1 & 26842 & 2.0 \\
\hline San Martín & 2824 & 1,3 & 24097 & 1.8 \\
\hline Loreto & 4260 & 1.9 & 23155 & 1.7 \\
\hline Tacna & 2951 & 1.3 & 22982 & 1.7 \\
\hline Ucayali & 2203 & 1.0 & 18518 & 1.4 \\
\hline Huánuco & 2121 & 0.9 & 17625 & 1.3 \\
\hline Ayacucho & 2230 & 1.0 & 14720 & 1.1 \\
\hline Tumbes & 1044 & 0,5 & 10290 & 0.8 \\
\hline Apurímac & 1044 & 0,5 & 9885 & 0.7 \\
\hline Madre de Dios & 916 & 0,4 & 9587 & 0.7 \\
\hline Moquegua & 2662 & 1,2 & 9518 & 0.7 \\
\hline Pasco & 2049 & 0,9 & 8555 & 0.6 \\
\hline Amazonas & 1431 & 0,6 & 7543 & 0.6 \\
\hline Huancavelica & $\mathbf{2 2 4 3 0 5}$ & $\mathbf{1 0 0 . 0}$ & $\mathbf{1 3 4 0 7 0 3}$ & $\mathbf{1 0 0 . 0}$ \\
\hline & & & & \\
\hline
\end{tabular}

Nota: El tamaño empresarial es determinado en base a la Ley $\mathrm{N}^{\circ} 30056$ 1/ Valores para el año 2011. 2/ El PBI está comprendido en la región Lima

Fuente: SUNAT, Registro Único del Contribuyente 2012

Elaboración: PRODUCE -Dirección de Estudios Económicos de MYPE e Industria (DEMI)

Esta distribución empresarial demuestra que Lima es la región que da soporte al crecimiento económico, lo cual genera un escenario de riesgo para la economía. Por ello, los IDH en las zonas que no son favorecidas con el desarrollo empresarial presentan problemas graves de crecimiento económico y por ende, la profundización de la pobreza, que no se va a corregir con los subsidios gubernamentales sino con inversiones reales y sostenidas en el tiempo. 


\section{CONCLUSIONES}

1. En la estructura empresarial en el país, las microempresas se constituyen en las más representativas, superando significativamente a las pequeñas y medianas empresas, situación que ha generado que se aperture un espacio laboral para una parte importante de la PEA a nivel nacional, pero este fenómeno se presenta con mayor fuerza en la ciudad de Lima; en especial, en las zonas de la costa que es en donde se concentran la mayor cantidad de estas empresas, situación que además, se refleja en la calidad de vida de la población.

2. En la región de la sierra como: Huancavelica, Apurímac, Pasco, entre otras zonas económicas, se aprecia la falta de presencia de unidades empresariales ya sean micro, pequeñas y medianas empresas, generándose escenarios de vulnerabilidad social como los síntoma de la pobreza y la migración. Igual caso se presenta en la región de la Selva, como Madre de Dios, que es otra región en la que no se ha desarrollado una cadena empresarial.

3. Las micro y pequeñas empresas, cualquiera sea su localización, están dedicadas mayormente al comercio y servicios, actividades que no generan mayores efectos multiplicadores en la economía.
Estas empresas focalizadas en estas actividades, son las que emplean la mayor cantidad de mano de obra, pero su contribución al crecimiento económico es limitada.

\section{REFERENCIAS BIBLIOGRÁFICAS}

1. INEI (2013) Perú: Estructura empresarial 2012. Lima - Perú.

2. INEI. Estadísticas sociales. Pobreza y gasto social. Lima. Perú.

3. KOTLER Philip (2005) Los diez pecados capitales del marketing. Gestión 2000.

4. MIRANDA G. (2005) Informalidad laboral y la acción estatal en el Perú: OEA Segunda Reunión de Grupo de Trabajo de la XIII Conferencia Interamericana de Ministros de Trabajo. Buenos Aires Argentina.

5. Ministerio de la Producción (2012) "Estadísticas de la micro, pequeña y mediana empresa. Lima Perú.

6. SALAZAR Carlos (2009) Evaluación y análisis de políticas públicas. Universidad Externado de Colombia.

7. www.produce.gob.pe

8. www.mintra.gob.pe 